\title{
Transesophageal Echocardiography for Ross-Konno Procedure
}

\author{
Shivani Aggarwal ${ }^{1}$ Probal Basumatary ${ }^{1} \quad$ V. Devagourou ${ }^{2}$ \\ ${ }^{1}$ Department of Cardiac Anaesthesiology, CTC, All India Institutes of \\ Medical Sciences, New Delhi, India \\ 2Department of CTVS, CTC, All India Institutes of Medical Sciences, \\ New Delhi, India

\begin{abstract}
Address for correspondence Shivani Aggarwal, Senior Resident, Department of Cardiac Anaesthesiology, CTC, All India Institutes of Medical Sciences, New Delhi 110029, India

(e-mail: drshivani.del@gmail.com).
\end{abstract}

\author{
Abstract \\ Keywords \\ - mild tricuspid \\ regurgitation \\ - mitral regurgitation \\ - moderate mitral \\ stenosis \\ - Ross-Konno procedure \\ - Ross procedure \\ - subvalvular disease \\ - transesophageal \\ echocardiography
}

Ross procedure involves replacing diseased aortic valve with pulmonary autograft and placing a pulmonary/aortic homograft between the right ventricle and pulmonary artery. In case of small aortic annulus in pediatric patients or in patients with multilevel left ventricular outflow obstruction, aortic annulus enlargement might be required concurrently. Konno type of aortic ventriculoplasty is most commonly done. The American Heart Association/American College of Cardiology (AHA/ACC) has classified transesophageal echocardiography (TEE) as class I indication for congenital heart disease

\section{Introduction}

Ross procedure involves replacing the diseased aortic valve with pulmonary autograft and coronary buttons and placing a pulmonary/aortic homograft between the right ventricle and pulmonary artery. In children with small aortic annulus or patients with multilevel left ventricular outflow obstruction, usually Konno type of aortic ventriculoplasty is added for aortic annular enlargement. In children and young adults, Ross-Konno procedure provides better hemodynamic profile, decreased need for anticoagulation, life expectancy similar to healthy age matched controls, and improved quality of life in the postoperative period. Concerns regarding complexity of procedure, the neo-aortic root dilatation disproportionate to somatic growth over time, progressive calcification, issues regarding durability of pulmonary valve (a valve from low pressure circulation implanted in a high-pressure circulation), and pulmonary homograft failure have limited the use of this procedure. Nevertheless, it cannot be denied that the living pulmonary valve provides better hemodynamic profile than bioprosthetic or mechanical valves.
Case: An 8-year-old boy with history of progressively increasing breathlessness on exertion (New York Heart Association [NYHA] II to III) was diagnosed with severe mitral regurgitation (MR), moderate mitral stenosis (MS) with subvalvular disease (SVD), and mild tricuspid regurgitation (TR) on transthoracic echocardiography (TTE). There was mild aortic stenosis (AS) with severe aortic regurgitation (AR). The pulmonary valve was of normal morphology with annular diameter of $13 \mathrm{~mm}$ whereas the aortic annular diameter was $9 \mathrm{~mm}$. On the basis of TTE, Ross-Konno procedure with mitral valve repair/replacement was planned. Intraoperative precardiopulmonary bypass (CPB) and transesophageal echocardiography (TEE) revealed severe MS ( - Fig. 1) and severe AR ( - Fig. 2a) with aortic annulus of $9 \mathrm{~mm}(\boldsymbol{- F i g}$. $\mathbf{2 b})$. The pulmonary valve was of normal morphology, but the annular diameter was measured as $19 \mathrm{~mm}$ ( - Fig. 3). On basis of TEE findings that were further confirmed by huge disparity in sizes of aorta and pulmonary artery on visual inspection, the Ross-Konno procedure was abandoned. Repair of aortic valve was attempted by decalcification and commissurotomy
Copyright @2018 Official Publication of The Simulation Society (TSS), accredited by International Society of Cardiovascular Ultrasound (ISCU).
License terms

(ㅇ) (1) $\Theta \circledast$ 

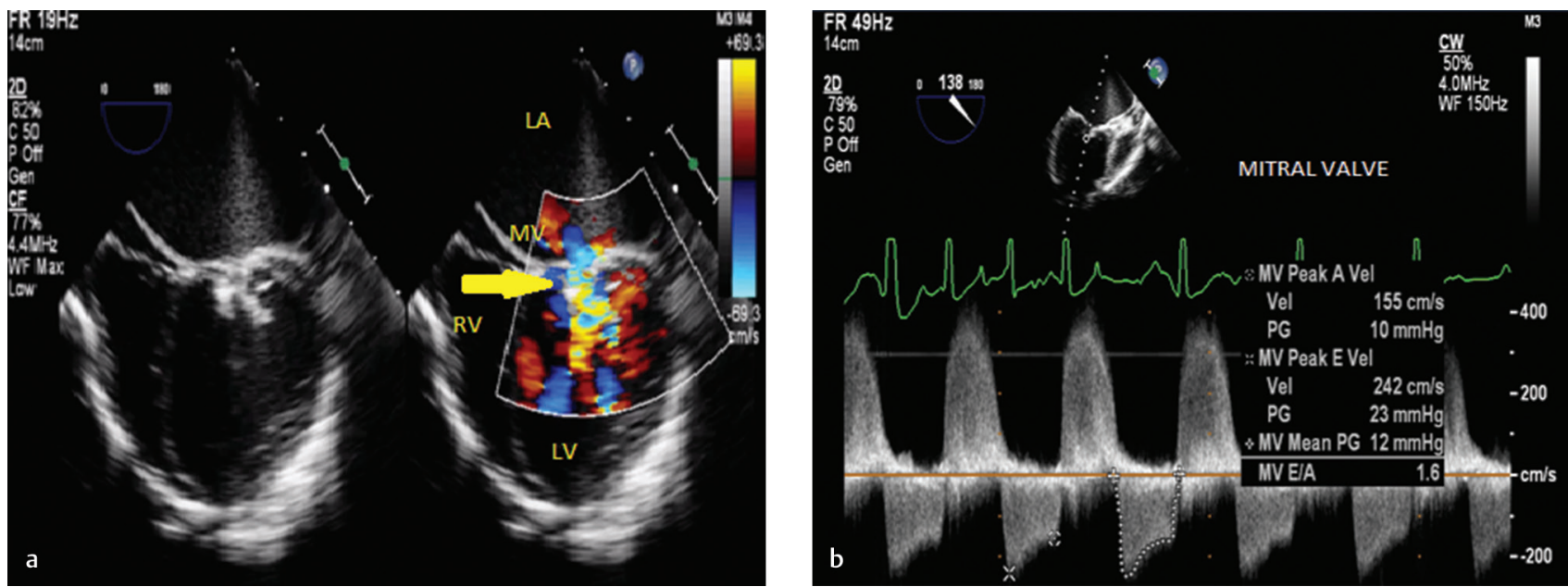

Fig. 1 (a) Midesophageal four-chamber view with color-flow Doppler showing color flow across mitral valve (yellow arrow) suggesting presence of mitral stenosis. The anterior and posterior mitral leaflets are thickened and heavily calcified. (b) Spectral Doppler across mitral valve (MV) in midesophageal aortic long-axis view showing gradients across MV. The peak diastolic gradient is $12 \mathrm{~mm} \mathrm{Hg}$ suggesting severe mitral stenosis. LA, left atrium; LV, left ventricle; RV, right ventricle.
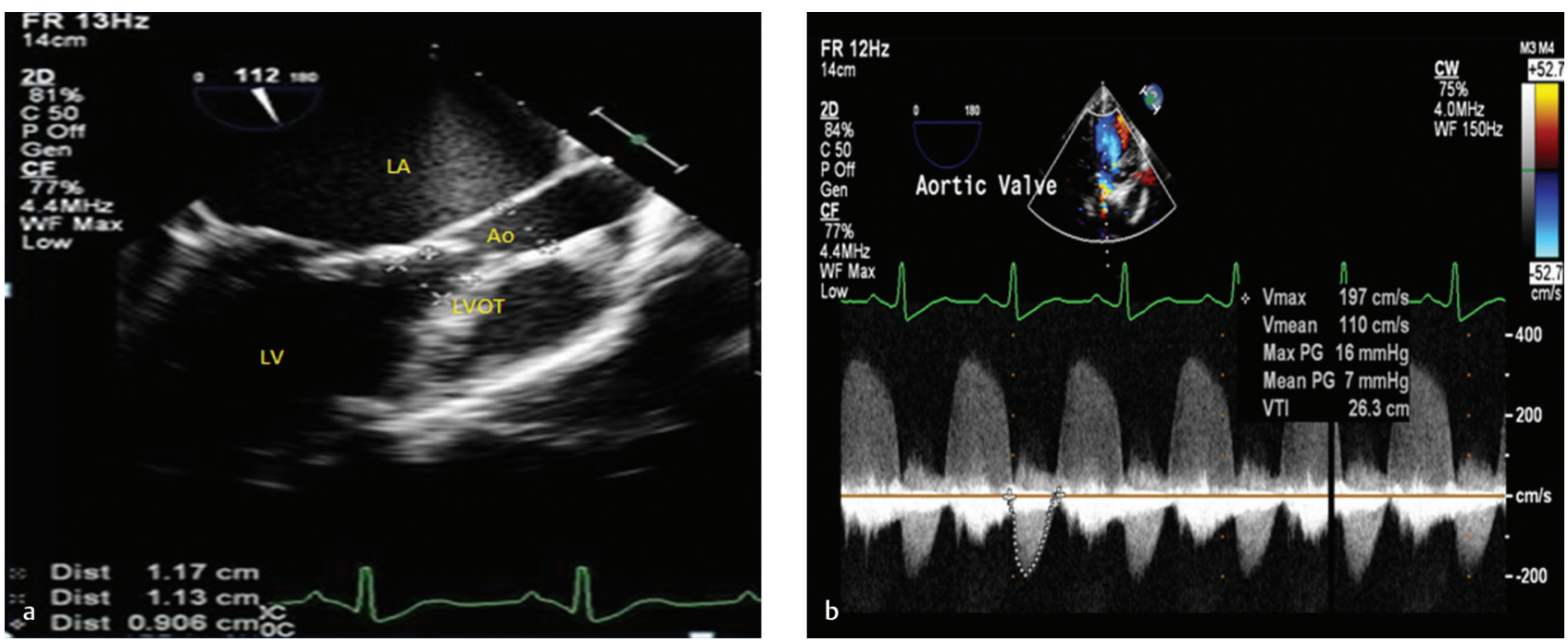

Fig. 2 (a) Midesophageal aortic long-axis view showing dimensions of left ventricular outflow tract (LVOT) 11.3 mm, aortic annulus (Ao) $9 \mathrm{~mm}$ and ascending aorta $11.7 \mathrm{~mm}$. (b) Spectral Doppler across aortic valve in deep transgastric view showing gradient across aortic valve. The mean systolic gradient is $7 \mathrm{~mm} \mathrm{Hg}$. There is severe aortic regurgitation as suggested by dense envelope in diastole.

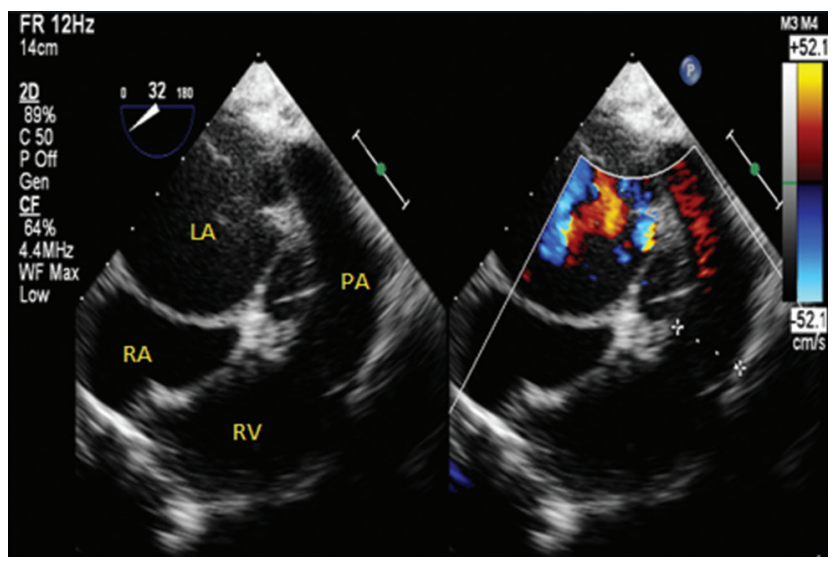

Fig. 3 Midesophageal modified RV inflow outflow view with colorflow Doppler showing pulmonary artery dimensions $19 \mathrm{~mm}$. whereas calcified anterior mitral leaflet and SVD mandated replacement of mitral valve. Following weaning from $\mathrm{CPB}$, TEE revealed successful repair of aortic valve with mild AR with peak systolic gradient $29 \mathrm{~mm} \mathrm{Hg}$ and mean systolic gradient $14 \mathrm{~mm} \mathrm{Hg}$ (-Fig. 4a, 4b).

\section{Discussion}

Transesophageal echocardiography is emerging as an indispensable tool for deciding intraoperative management of complex congenital cardiac lesions. The use of intraoperative TEE may change the entire plan of surgical management as happened in this case. The basic prerequisite for Ross procedure is that the pulmonary annulus should be at most within $5 \mathrm{~mm}$ of aortic annulus and 

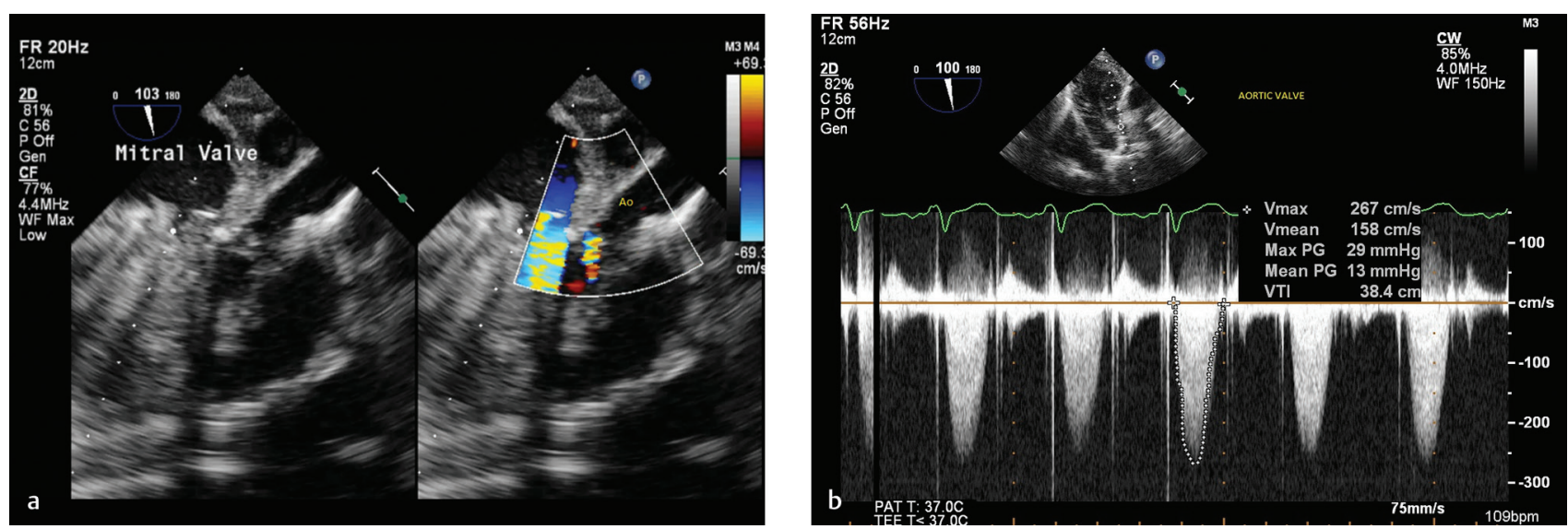

Fig. 4 (a) Midesophageal aortic valve long axis view with color flow Doppler showing mild AR in postoperative period following aortic valve repair. (b) Spectral Doppler across aortic valve in deep TG view in postoperative period following aortic valve repair. The peak systolic gradient is $29 \mathrm{~mm} \mathrm{Hg}$ and mean systolic gradient is $13 \mathrm{~mm} \mathrm{Hg}$.

should have normal morphology. The TEE in this case although revealed a normal morphologic pulmonary valve, there was huge disparity in the annuli of aorta and pulmonary artery, which changed the entire surgical plan. The Ross-Konno procedure had to be abandoned, and decision to repair the aortic valve was taken. In the post-CPB period, the aortic valve assessment revealed mild AR that was accepted because the child was only 8 years old.

Transesophageal echocardiography is recommended as a class I indication for congenital heart disease ${ }^{1}$ by the American Heart Association/American College of Cardiology (AHA/ACC) affecting intraoperative management ${ }^{2}$ as well as postoperative prognosis. Stevenson et al have demonstrated that among congenital disorders, Ross procedure requires most evaluation with intraoperative TEE. ${ }^{3}$ TEE is required in pre-CPB period to confirm the diagnosis; identify the mechanism of aortic pathology; assess morphology of the pulmonary valve and presence of pulmonary hypertension; and measure aortic and pulmonary annulus $( \pm 3 \mathrm{~mm}$ ), any sub- or supra-aortic left ventricular outflow obstruction, and right ventricular outflow obstruction. In addition, TEE helps in assessment of volume status, ventricular function, and cardiac output that is helpful in intraoperative management. In the post-CPB period, TEE examination should be aimed at assessment of any anatomic/functional derangement of autograft and allograft (leaflet coaptation/prolapsed/restriction), transvalvular gradient across both autograft and allograft, any regional wall motion abnormality in region of the left main coronary artery or left anterior descending artery or septal perforators, and presence of any new aortic insufficiency.
Neo-aortic insufficiency following Ross-Konno procedure predicts increased likelihood of progression to hemodynamically significant aortic regurgitation on long-term follow-up requiring reoperation.

Thus, TEE is becoming an important tool in the armamentarium of cardiac anesthesiologist for guiding intraoperative management, especially in complex congenital heart disease. TEE helps in evaluating suitability of valve for repair, and especially in delineating any residual lesion amenable to repair after the surgical procedure. It results in improved patient prognosis and reduced morbidity and mortality in the postoperative period.

\section{Conflict of Interest}

None.

\section{References}

1 Cheitlin MD, Armstrong WF, Aurigemma GP, et al. ACC/ AHA/ASE 2003 guideline update for the clinical application of echocardiography-summary article: a report of the American College of Cardiology/American Heart Association Task Force on Practice Guidelines (ACC/AHA/ASE Committee to Update the 1997 Guidelines for the Clinical Application of Echocardiography). J Am Coll Cardiol 2003;42(5):954-970

2 Guzeltas A, Ozyilmaz I, Tanidir C, et al. The significance of transesophageal echocardiography in assessing congenital heart disease: our experience. Congenit Heart Dis 2014;9(4): 300-306

3 Stevenson JG, Sorensen GK, Gartman DM, Hall DG, Rittenhouse EA. Transesophageal echocardiography during repair of congenital cardiac defects: identification of residual problems necessitating reoperation. J Am Soc Echocardiogr 1993;6(4): 356-365 